\title{
AVALIAÇÃO DA MECÂNICA VENTILATÓRIA EM INDIVÍDUOS COM DISFUNÇÃO TÊMPORO-MANDIBULAR E ASSINTOMÁTICOS
}

\author{
Pasinato $\mathrm{F}^{1}$, Corrêa ECR ${ }^{2}$ e Peroni $\mathrm{ABF}^{2}$ \\ ${ }^{1}$ Fisioterapeuta \\ ${ }^{2}$ Departamento de Fisioterapia, Centro de Ciências da Saúde, Universidade Federal de Santa Maria, Santa Maria, SC - \\ Brasil \\ Correspondência para: Fernanda Pasinato, Rua Dr. Bozano, 696, apto 303, CEP 97015-000, Santa Maria, RS - Brasil, \\ e-mail: fepasinato2002@yahoo.com.br
}

Recebido: 27/09/2005 - Aceito: 12/05/2006

\begin{abstract}
RESUMO
Contexto: A relação entre disfunções respiratórias e têmporo-mandibulares tem sido recentemente constatada pela incidência de dor orofacial entre indivíduos com história de doenças respiratórias obstrutivas tanto de vias aéreas superiores como nas inferiores. Objetivo: Avaliar comparativamente a mecânica ventilatória em indivíduos com disfunção têmporo-mandibular (DTM) e em indivíduos assintomáticos. Método: Participaram do estudo 35 voluntários, sendo 20 indivíduos com DTM e 15 assintomáticos. Os participantes foram avaliados para verificação da presença e grau de disfunção têmporo-mandibular. Realizouse avaliação respiratória, por meio de inspeção do padrão e tipo ventilatório, medida dos perímetros torácicos, teste de força dos músculos respiratórios e histórico de enfermidade respiratória. Resultados: Nos indivíduos com DTM, o modo respiratório bucal (30\%) e misto (15\%), bem como o padrão ventilatório apical (45\%) foram predominantes. Observou-se uma menor expansibilidade torácica e uma redução estatisticamente significante no índice de amplitude abdominal nos indivíduos com DTM. Estes indivíduos também apresentaram maior incidência de patologias respiratórias obstrutivas tanto de vias aéreas superiores (70\%) como inferiores (45\%) em relação aos assintomáticos. Conclusão: Os resultados indicam que existe uma influência da mecânica ventilatória sobre a ocorrência de disfunção têmporo-mandibular.
\end{abstract}

Palavras-chave: ventilação pulmonar, transtornos da articulação têmporo-mandibular, mastigação, postura.

\section{ABSTRACT \\ Evaluation of Ventilatory Mechanics in Individuals with Temporomandibular Dysfunction and in Asymptomatic Individuals}

Background: The relationship between respiratory and temporomandibular dysfunctions has recently been brought to light through the incidence of orofacial pain among individuals with a history of obstructive respiratory diseases in the upper and lower airways. Objective: To comparatively evaluate the ventilatory mechanics in individuals with temporomandibular dysfunction (TMD) and in asymptomatic individuals. Method: Thirty-five volunteers took part in the study: 20 individuals with TMD and 15 asymptomatic individuals. The participants were evaluated to verify the presence and degree of TMD. A respiratory evaluation was carried out by means of inspecting the ventilatory pattern and type, measuring the chest perimeter, testing the respiratory muscle strength and investigating the history of respiratory diseases. Results: The mouth (30\%) and mixed (15\%) breathing types and the apical ventilatory pattern (45\%) predominated in individuals with TMD. There was less chest expandability and a statistically significantly smaller abdominal amplitude index in individuals with TMD. These individuals also presented higher incidence of obstructive respiratory diseases in the upper (70\%) and lower (45\%) airways, in relation to the asymptomatic individuals. Conclusion: The results indicate that there is an influence from ventilatory mechanics on the occurrence of temporomandibular dysfunction.

Key words: lung ventilation, temporomandibular joint disorders, mastication, posture. 


\section{INTRODUÇÃO}

As disfunções têmporo-mandibulares (DTMs) compreendem uma ampla categoria de desordens que engloba alterações clínicas na musculatura mastigatória, na articulação têmporo-mandibular (ATM), ou em ambas ${ }^{1}$.

Porém, alterações mais globais também parecem estar envolvidas na etiologia das DTMs, especialmente a postura corporal e, mais recentemente, a influência da mecânica e padrão ventilatórios nas DTMs vêm despertando o interesse de alguns pesquisadores ${ }^{2,3,4}$.

A relação entre respiração e DTM é determinada especialmente pelo uso excessivo da musculatura inspiratória acessória, o que pode também acarretar alterações posturais. Fatores como: padrão ventilatório apical, tensão/ansiedade e respiração bucal podem ser indicados como os principais responsáveis por estas alterações ${ }^{4}$.

A retração da cadeia inspiratória que eleva o tórax e impede-o de voltar à posição expiratória inicial, limita conseqüentemente os movimentos do diafragma. Além disso, o encurtamento dos músculos acessórios tônicos antigravitários causa uma série de alterações posturais na coluna cervical, ombros e dorso ${ }^{3}$.

Rocabado ${ }^{5}$ considerou a respiração costal superior, curta e insuficiente e a respiração bucal como sintomas apresentados por pacientes com DTM. Outros autores ${ }^{6,7}$ consideram que a respiração curta leva ao desenvolvimento da postura de cabeça anteriorizada com estimulação da rotação cranial posterior. Estudos recentes sobre DTM vêm abordando a avaliação do padrão ventilatório e modo respiratório (nasal ou bucal) $)^{2,8}$.

De acordo com Ribeiro9 ${ }^{9}$ o músculo esternocleidomastóideo (ECM) é um dos principais responsáveis pelos transtornos disfuncionais da cabeça e pescoço associados com disfunção do aparelho estomatognático. A contração bilateral do ECM causa flexão anterior da coluna cervical sobre a torácica e anteriorização da cabeça. Isto pode aumentar o esforço inspiratório, contribuindo para um padrão ventilatório apical, e por sua vez, um maior recrutamento dos músculos acessórios da inspiração. A anteriorização da cabeça também tensiona a musculatura hióidea, tracionando a mandíbula posteriormente.

Com base na literatura consultada, este estudo teve como objetivo avaliar a mecânica e padrão ventilatório de indivíduos com DTM e indivíduos assintomáticos. Além disso, o estudo propôs-se a aprofundar o conhecimento sobre este tema ainda pouco explorado e fornecer suporte teórico à prática clínica para uma abordagem global do paciente portador desta disfunção.

\section{METODOLOGIA}

Este estudo foi submetido e aprovado pelo Comitê de Ética em pesquisa da Universidade Federal de Santa Maria
(016848/2005). Os voluntários foram informados a respeito dos objetivos e procedimentos utilizados na pesquisa e assinaram um termo de consentimento livre e esclarecido.

Foram estudados 35 voluntários, com idades entre 19 e 53 anos, distribuídos em 2 grupos: grupo DTM e grupo assintomático.

Grupo DTM: 20 sujeitos selecionados entre indivíduos que procuraram o Serviço de Odontologia da Universidade Federal de Santa Maria com sintomas de dor orofacial ou na articulação têmporo-mandibular, com DTM confirmada pelo diagnóstico odontológico. Dezenove sujeitos eram do gênero feminino e um do masculino, com idades entre 20 a 46 anos, e média aproximada de 27 anos ( $\mathrm{DP}=7,05)$.

Foram excluídos do estudo indivíduos com histórico de traumas ortopédicos na região facial, doença reumática, doença pulmonar crônica, pacientes em tratamento odontológico com uso de placa (miorrelaxante ou reposicionadora) com remissão de sintomas ou em tratamento fisioterápico ou medicamentoso específico.

Grupo Assintomático: 15 sujeitos classificados através do Î́ndice Anamnésico de Fonseca ${ }^{10}$ quanto ao grau de disfunção como sem disfunção ou com disfunção leve (assintomáticos). Este índice foi utilizado com a finalidade de triagem dos sujeitos assintomáticos por ser de fácil e rápida aplicação ${ }^{2}$. Os voluntários eram todos do gênero feminino e possuíam idades entre 19 e 53 anos e média aproximada de 24 anos ( $\mathrm{DP}=8,24)$.

Após o diagnóstico odontológico de DTM, os indivíduos com disfunção foram submetidos ainda à avaliação pelo Índice Anamnésico e Clínico de Disfunção e Estado Oclusal ${ }^{11}$, que inclui: (1) Índice de Disfunção Clínica, que avalia a amplitude e presença de desvio no movimento mandibular, presença de sons na ATM (verificada através da auscultação e palpação articular), sensibilidade à palpação dos músculos mastigatórios e da ATM; (2) Índice de Mobilidade articular, verificando a medida das amplitudes máximas de movimento mandibular em abertura, lateralidade direita e esquerda e protrusão (medidas realizadas por meio de paquímetro - Figura 1); (3) Índice Anamnésico de Disfunção, que avalia sintomas relatados pelos pacientes tais como sons na ATM, sensação de fadiga na mandíbula, sensibilidade ou rigidez na mandíbula ao acordar ou em sua abertura, dificuldade para abrir a boca em toda sua amplitude, travamento, luxação, dor ao movimento, dor na ATM ou músculos mastigatórios; (4) Índice de Estado Oclusal (avaliado por odontólogo especializado na área), verificando o número de dentes e sua oclusão, bem como a presença de interferências articulares ou oclusais.

A avaliação respiratória dos participantes do estudo contemplou os seguintes aspectos: história de patologias respiratórias atual e/ou prévia, padrão ventilatório, modo respiratório e mobilidade torácica por meio do Índice de Amplitude Tóraco-abdominal (IATA) com medida dos perímetros torácicos (nos níveis axilar, xifoidal e umbilical). O IATA consiste no valor ponderado entre as medidas obtidas 
com a fita métrica na inspiração e expiração máximas. É um modo simples e acessível na prática clínica de mensurar a expansibilidade tóraco-abdominal de indivíduos de diferentes dimensões cirtométricas ${ }^{12}$. O exame clínico da força muscular respiratória foi realizado segundo orientação de Cuello ${ }^{13}$.

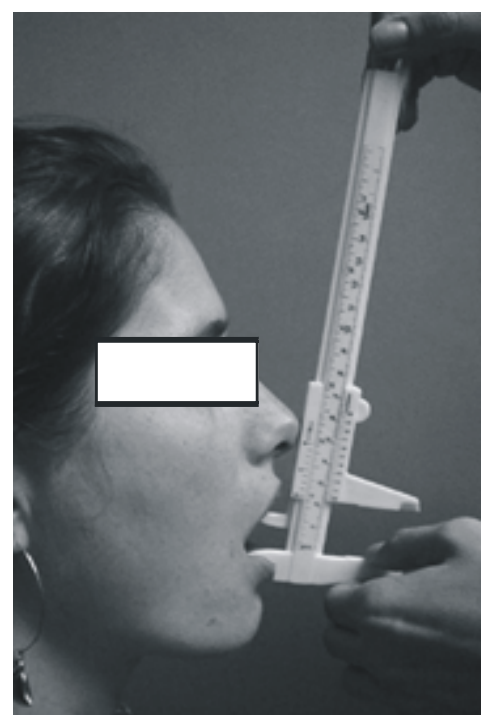

Figura 1. Avaliação da amplitude de abertura da boca com uso de paquímetro.

Após a coleta dos dados, os mesmos foram submetidos à análise estatística descritiva, com cálculo da média aritmética e desvio padrão dos valores obtidos nos referidos grupos. Para análise comparativa dos resultados quanto à expansibilidade tóraco-abdominal, foi utilizado teste "t" de Student para variáveis independentes (software Statistica 5.5).

\section{RESULTADOS}

Inicialmente, serão apresentados os resultados relacionados ao grau de disfunção, obtidos pelo Índice de Helkimo ${ }^{13}$ que demonstram o perfil dos participantes em relação à DTM. Verificou-se, no Índice de Disfunção Clínica (IDC), um percentual de $40 \%$ e $45 \%$ dos indivíduos com sinais e sintomas de DTM leve e moderada, respectivamente. Quanto ao Índice de Mobilidade Articular (IMA), foi verificado 30\% dos indivíduos com mobilidade articular normal, 65\% com disfunção leve e 5\% com disfunção severa. No Índice Anamnésico de Disfunção (IAD) 60\% e 40\% com disfunção severa e leve, respectivamente. No Índice de Estado Oclusal (IEO) foi observado disfunção moderada em 65\%, disfunção leve em 25\% e ausência de disfunção em 10\% dos indivíduos.

Com relação à avaliação respiratória, houve maior incidência de doenças obstrutivas das vias aéreas superiores (70\%) e das vias aéreas inferiores (45\%) em indivíduos com
DTM comparado aos assintomáticos (40\% e 7\% com doenças de vias aéreas superiores e inferiores, respectivamente). Dentre as patologias citadas constaram rinite alérgica, sinusite, desvio de septo nasal e adenóides hipertróficas.

Quanto ao tipo respiratório, observou-se em ambos os grupos estudados um predomínio da respiração nasal (55\% no grupo DTM e 93\% no grupo assintomático). Já o tipo respiratório bucal não foi observado entre os sujeitos do grupo assintomático e apresentou-se em 30\% dos indivíduos com DTM. O tipo respiratório misto, no qual o indivíduo alterna os tipos nasal e bucal, foi verificado em 15\% dos indivíduos com DTM e em apenas 7\% dos assintomáticos (Tabela 1).

A Tabela 1 ilustra também a distribuição dos participantes quanto ao padrão ventilatório. O padrão ventilatório apical predominou nos indivíduos com DTM. O padrão ventilatório misto apresentou-se em 25\% e 47\% dos indivíduos com DTM e assintomáticos, respectivamente. O padrão ventilatório diafragmático foi predominante no grupo assintomático.

Pode-se observar na Tabela 2, que, em média, os indivíduos do grupo assintomático obtiveram maiores índices de expansibilidade torácica em todos os níveis, quando comparados aos indivíduos com DTM. Observou-se também uma maior expansibilidade a nível supra-umbilical nos sujeitos assintomáticos, com uma diferença estatisticamente significante entre os grupos $(p=0,016)$.

Com relação à avaliação da força muscular (Tabela 3), apenas um indivíduo com distúrbios na ATM obteve força máxima do músculo diafragma (4), 65\% regular, 25\% fraco e um indivíduo apresentou vestígio de contração. A força máxima foi apresentada por 53\% dos indivíduos assintomáticos neste teste.

\section{DISCUSSÃO}

Para avaliação da ATM foram empregados os Índices de Fonseca e Helkimo, sendo que o primeiro teve como finalidade classificar os voluntários em indivíduos com DTM e assintomáticos e o segundo para o exame detalhado da articulação, oclusão e músculos mastigatórios. Estudos prévios utilizaram estes instrumentos para a identificação de fatores etiológicos e o acometimento multifatorial de pacientes com DTM e demonstraram correlação entre estes índices ${ }^{2}$.

Os resultados apresentados sugerem uma possível relação entre a incidência de patologias respiratórias e DTM, já que estas prevaleceram nos indivíduos com disfunção têmporo-mandibular. Esta relação corrobora com os achados da literatura revisada, que referem que a obstrução nasal é motivo de alterações na postura craniocervical e mandibular para facilitar a respiração ${ }^{4}$. Ainda, Ribeiro ${ }^{9}$ verificou maior atividade eletromiográfica dos músculos ECM e trapézio durante a inspiração nasal de crianças respiradoras bucais do que em respiradoras nasais, conseqüência do maior esforço da musculatura acessória da respiração para vencer a resistência aumentada das vias aéreas superiores. 
Tabela 1. Tipo e Padrão Respiratório apresentado por indivíduos com DTM e assintomáticos (\%).

\begin{tabular}{lllc}
\hline & & Grupo DTM (\%) & Grupo Assintomático (\%) \\
\hline Tipo Respiratório & Nasal & 55 & 93 \\
& Bucal & 30 & 0 \\
& Misto & 15 & 7 \\
\multirow{2}{*}{ Padrão Respiratório } & Diafragmático & 30 & 40 \\
& Apical & 45 & 13 \\
& Misto & 25 & 47 \\
\hline
\end{tabular}

Tabela 2. Média e Desvio Padrão dos Índices de Amplitude Tóraco-abdominal (IATA) de indivíduos com DTM e assintomáticos.

\begin{tabular}{lccc}
\hline \multicolumn{1}{c}{ Cirtometria } & Grupo DTM(x $\pm \mathbf{d p})$ & Grupo Assintomático $(\mathbf{x} \pm \mathbf{d p})$ & $\mathbf{p}$ \\
\hline Axilar & $5.139 \pm 2.09$ & $6.21 \pm 1.94$ & 0.1352 \\
Xifoidal & $5.021 \pm 2.35$ & $6.47 \pm 2.22$ & 0.0766 \\
Supra-umbilical & $4.3 \pm 2.53$ & $6.79 \pm 3.19$ & $0.016^{*}$ \\
\hline${ }^{*} \mathrm{p}<0.05$ & & &
\end{tabular}

Tabela 3. Distribuição relativa indivíduos com DTM e assintomáticos quanto ao grau de força muscular respiratória (\%).

\begin{tabular}{lcccccccccc}
\hline & \multicolumn{1}{c}{ Grupo DTM (\%) } & \multicolumn{1}{c}{ Grupo Assintomático (\%) } \\
\hline Força Muscular & $\mathbf{4}$ & $\mathbf{3}$ & $\mathbf{2}$ & $\mathbf{1}$ & $\mathbf{0}$ & $\mathbf{4}$ & $\mathbf{3}$ & $\mathbf{2}$ & $\mathbf{1}$ & $\mathbf{0}$ \\
Diafragma & 5 & 65 & 25 & 5 & 0 & 53 & 33 & 13 & 0 & 0 \\
Intercostais Inferiores & 35 & 50 & 10 & 5 & 0 & 33 & 67 & 0 & 0 & 0 \\
Intercostais Superiores & 35 & 55 & 10 & 0 & 0 & 47 & 47 & 7 & 0 & 0 \\
& & & & & & & & & & \\
\hline
\end{tabular}

Na avaliação do tipo respiratório, observou-se que o bucal e misto foram mais prevalentes nos indivíduos com DTM. No grupo assintomático, não foi encontrado nenhum indivíduo que adotasse a respiração do tipo bucal. Além disso, 50\% dos indivíduos com DTM e respiração bucal apresentaram índice de estado oclusal severos. Estes resultados sugerem a influência do tipo de respiração adotada (respiração bucal) pelos indivíduos no desenvolvimento de distúrbios da ATM. A respiração bucal já foi descrita como fator determinante de dor craniofacial e maloclusão, devido à hiperatividade do músculo ECM que produz uma anteriorização da cabeça, com tensão nos músculos supra e infra-hióideos, descida da língua e rotação posterior da mandíbula ${ }^{4}$. Chaves et al. ${ }^{14}$ confirmam a constatação de que as recorrentes infecções das vias aéreas superiores em crianças asmáticas determinam obstrução nasal associada à respiração bucal que, por sua vez, relaciona-se com o surgimento de significativas alterações craniofaciais. O estudo sobre a incidência de disfunção têmporo-mandibular em crianças asmáticas com respiração bucal mostrou que todas as crianças apresentavam DTM, em sua maioria de severidade relevante. Entre os indivíduos com DTM e tipo respiratório bucal, 50\% apresentaram índices de Estado Oclusal ${ }^{11}$ severos, e outros participantes que também apresentaram este índice na avaliação oclusal apresentaram história de respiração bucal e/ou doença respiratória da via aérea inferior na infância.

Observou-se também um predomínio do padrão ventilatório apical nos indivíduos com DTM em relação aos indivíduos assintomáticos. Estes resultados evidenciam a relação funcional entre a ATM e a coluna cervical. Ainda, 
relatos descrevem a respiração costal superior, curta e insuficiente, e a respiração bucal como sintomas apresentados por pacientes com DTM, associada à postura de cabeça anteriorizada com pescoço e dorso rígidos ${ }^{2,7,8,15}$.

Foi constatada, em média, uma menor expansibilidade torácica nos indivíduos com DTM, em todos os níveis pesquisados, sendo verificado um menor (estatisticamente significante) índice de amplitude abdominal nos indivíduos portadores de DTM. Este resultado indica que a maior amplitude de movimento abdominal em indivíduos assintomáticos está possivelmente associada a um padrão respiratório mais adequado, com predomínio do trabalho diafragmático, enquanto os indivíduos com DTM tendem a apresentar padrões costal e apical.

Farah e Tanaka ${ }^{16}$ avaliaram a mobilidade do tronco de indivíduos com DTM através de medidas de flexibilidade ao movimento do tronco (flexão e rotação) e expansibilidade do tórax com medidas cirtométricas. Similar a este estudo, as autoras também encontraram importante diminuição da expansibilidade torácica nos três níveis pesquisados e redução da amplitude de movimento da coluna cervical, resultante do encurtamento seqüencial das cadeias musculares posterior e respiratória (músculos escalenos e ECM) alcançando até a cadeia ântero-medial do quadril.

A força muscular respiratória dos voluntários do estudo foi avaliada através do método clínico descrito por Cuello ${ }^{15}$, que embora possua um caráter subjetivo intrínseco, foi o único encontrado na literatura consultada para avaliar separadamente os músculos responsáveis pela respiração.

Os indivíduos com DTM apresentaram menores graus de força muscular diafragmática em relação a indivíduos assintomáticos, indicando que a desvantagem diafragmática pode levar ao uso mais intenso da musculatura acessória da respiração, favorecendo o surgimento de distúrbios dolorosos craniofaciais. Segundo Souchard ${ }^{17}$, em condições normais o diafragma é o principal músculo responsável pela respiração automática de pequena amplitude. Quando existe a necessidade de aumentar o volume das trocas gasosas, a contração diafragmática deve ser intensificada e, somente após isso, entram em ação os músculos inspiratórios acessórios.

\section{CONCLUSÃO}

O modo respiratório bucal e misto e o padrão ventilatório apical predominaram em indivíduos com DTM, sendo que, nos indivíduos assintomáticos, os padrões ventilatórios tendiam a ser misto e diafragmático.

Foi constatada, em média, uma menor mobilidade/ expansibilidade torácica dos portadores de DTM, em todos os níveis pesquisados.

Os indivíduos com DTM apresentaram menores graus de força muscular diafragmática em relação aos sujeitos assintomáticos.
Agradecimentos: Ao Prof. Dr. Claudio Figueiró da Universidade Federal de Santa Maria, responsável pela avaliação odontológica dos participantes do estudo.

\section{REFERÊNCIAS BIBLIOGRÁFICAS}

1. Okeson JP. Dor orofacial: guia de avaliação, diagnóstico e tratamento. 4ª ed. São Paulo: Quintessence; 1998.

2. Oliveira AS. Caracterização multifatorial de uma população de portadores de desordens temporomandibulares [tese]. Piracicaba: FOP/UNICAMP; 2002.

3. Neves AO, Caetano VC. A relação entre desordem temporomandibular, postura corporal e músculo diafragma na visão da fisioterapia: caso clínico. Rev. Serviço ATM 2004; 4(1):35-39.

4. Corrêa ECR, Bérzin F. Temporomandibular disorder and dysfunctional breathing. Braz J Oral Sci 2004; 3(10):498-502.

5. Rocabado M. Material didático do curso Avances internacionales en biomecanica articular; Centro de Estudios de las Disfunciones Musculoesqueleticas, Módulos I e II, Novembro/2003 e Julho/ 2004, São Paulo/SP.

6. Hruska RJ. Influences of dysfunctional respiratory mechanics on orofacial pain. Dent Clin North Am 1997; 4(20):211-227.

7. Dutton M, Mark D. Manual therapy of the spine: an integrated approach. McGraw-Hill Medical Publishing Division; 2002.

8. Pereira Jr FJ, Huggins KH, Dworkin SF. Critérios de diagnóstico em pesquisa das desordens temporomandibulares. RDC/DTM. [cited 2004 Aug 10]. Available from URL: http://rdctmdinternational.org/tranlations/RDC-Portuguese.pdf

9. Ribeiro EC. Estudo eletromiográfico dos músculos esternocleidomastóideo e trapézio em crianças respiradoras bucais e nasais [dissertação]. Santa Maria: Univ. Federal de Santa Maria; 2000.

10. Fonseca DM. Disfunção craniomandibular (DCM): diagnóstico pela anamnese [dissertação]. Bauru: FOB-USP; 1994.

11. Helkimo M. Studies a function and dysfunction of the mastigatory system - II Index for anamnesic and clinical dysfunction and oclusal state. Swet Dent J. 1974; 67(2):101-121.

12. Jamami M, Pires VA, Costa D. Efeitos da intervenção fisioterápica na reabilitação pulmonar de pacientes com doença pulmonar obstrutiva crônica. Rev. Fisioter. Univ. São Paulo 1999; 6 (2):140-153.

13. Cuello A. Kinesiologia neumo cardiologica. Buenos Aires: Silka; 1980.

14. Chaves TC, Aguiar DN, Costa D, Bevilacqua-Grossi D. Aplicação do Índice de Disfunção Crânio-mandibular (IDCM) em crianças asmáticas. Rev Bras de Fisioter 2002: 6 Suppl: 67.

15. Rocabado M. Cabeza y cuello - tratamiento articular. Buenos Aires: Intermédica; 1979.

16. Farah EA, Tanaka CR. Postura e mobilidade da coluna cervical e do tronco em portadores de alterações miofuncionais orais. Rev APCD 1997; 51(2):171-175.

17. Souchard Phe. Respiração. São Paulo: Summus; 1989. 